Journal of Environmental
ISSN: $2525-815 \mathrm{X}$

\title{
Vegetação de tabuleiro: diversidade de espécies lenhosas em áreas da Paraíba, Nordeste do Brasil
}

\section{Forest on tertiary "tabuleiro": diversity of woody species in areas of Paraíba state, Northeast Brazil}

\author{
Carmen Sílvia Zickel ${ }^{\mathrm{a}}$, Adriano Vicente ${ }^{\mathrm{a}}$, Luciana Belfort ${ }^{\mathrm{b}}$, Catherine Rios Santos ${ }^{\mathrm{a}}$, Eduardo Bezerra \\ de Almeida Jr. ${ }^{\text {b }}$ \\ ${ }^{a}$ Universidade Federal Rural de Pernambuco-UFRPE, Programa de Pós-graduação em Botânica-PPGB. Av. Dom Manoel \\ de Medeiros, s/n, Dois Irmãos, Recife-PE, Brasil. CEP: 52171-900. E-mail: zickelbr@yahoo.com, \\ adrvicente@pop.com.br, catherineriosantos@gmail.com. \\ b Universidade Federal do Maranhão-UFMA, Programa de Pós-Graduação em Biodiversidade e Conservação-PPGBC. \\ Av. dos Portugueses, s/n, Bacanga, São Luís-MA, Brasil. CEP: 65085-580. E-mail: lucibelfort@ hotmail.com, \\ ebaj25@yahoo.com.br.
}

\begin{tabular}{l}
\hline A R T I C L E I N F O \\
Recebido 17 Jul 2020 \\
Aceito 25 Ago 2021 \\
Publicado 07 Out 2021
\end{tabular}

\begin{abstract}
A B S T R A C T
Coastal forest on tertiary, known as "tabuleiro" is a local term used to define areas of open vegetation, which is distributed in sandy soils. The study compares woody species' structure, richness, and diversity from four "tabuleiro" areas in Paraíba state, Northeastern Brazil. For structural analysis of the vegetation, the quadrant point method was used, being installed five transects with 10 points each. At each point, individuals with perimeter at ground height $>10 \mathrm{~cm}$ were analyzed. In the four areas studied (Mamanguape, João Pessoa, Conde, and Pedras de Fogo) 82 species were registered, in 29 families. Variations were found in total density, diversity, mean height and diameter, total basal área, and proportion of individuals with tillers. Differences in height frequency distribution were also recorded. The diversity index $\left(H^{\prime}\right)$ values ranged from 2.44 to 3.37 (Mamanguape, and João Pessoa, respectively); and the equability index $\left(\mathrm{J}^{\prime}\right)$ ranged from 0.68 to 0.88 (Pedras de Fogo, and João Pessoa, respectively). From the data presented, the flora of the "tabuleiro" areas varied in the diversity and spatial structure of the vegetation, despite sharing similar environmental conditions. The influence of the surrounding areas, the edaphic factors, and the different degrees of disturbance in the floristic composition may explain the heterogeneity found in the "tabuleiro" areas.
\end{abstract}

Keywords: Coastal vegetation, phytosociology, woody structure. 
os diferentes graus de perturbação na composição florística podem explicar a heterogeneidade encontrada nas áreas de tabuleiros.

Palavras-Chave: Vegetação costeira, Fitossociologia, estrutura lenhosa.

\section{Introdução}

O tabuleiro apresenta-se distribuído em manchas, dentro e no contorno da floresta atlântica, com vegetação esparsa, arbustos ramificados e baixos, ocorrendo em locais onde os solos apresentam textura arenosa, datados no Terciário, pertencente ao Grupo Barreiras (Andrade-Lima, 1954; Tavares, 1964; Bigarella, 1975). Apesar dessa complexidade, a vegetação de tabuleiro diferencia-se por se desenvolver na região litorânea do Nordeste do Brasil, recobrindo os baixos platôs formados por sedimentos terciários do Grupo Barreiras (Tavares, 1960; Salgado et al., 1981).

A vegetação de tabuleiro costeiro apresenta fisionomia semelhante tanto com o cerrado sensu stricto (Tavares 1960, 1964), quanto com a vegetação de restinga (Oliveira-Filho, 1993; Santos-Filho \& Zickel, 2013), devido a presença de muitas espécies características deste bioma (Andrade-Lima, 1960). E essa "igualdade" com o cerrado se dá mais pela presença de espécies comuns do que por sua fisionomia (Andrade-Lima, 1970). Andrade-Lima (1960) sugeriu ainda que algumas regiões próximas ao litoral, como os campos de restinga, podem ser um estágio intermediário para os tabuleiros, devido à presença de espécies em comum aos dois ambientes (Andrade-Lima, 1960). Todavia, Oliveira-Filho (1993) sugeriu que a vegetação de tabuleiro, em algumas regiões, pode ocorrer adjacente à restinga formando um gradiente vegetacional. Ainda, segundo o autor, a fisionomia das áreas de contato entre restinga e tabuleiro é heterogênea, predominando a floresta aberta, com muitas clareiras e inclusões de cerrado arborizado, surgidas provavelmente com a contribuição do fogo e do corte.

Apesar da importância econômica e ecológica dos tabuleiros, são poucos os estudos voltados para esse ecossistema, e os que existem estão concentrados na região sudeste do Brasil (Sousa \& Santos-Filho, 2020). Dessa forma, as diferentes descrições da fisionomia dos tabuleiros provêm da falta de estudos sobre aspectos de sua estrutura e heterogeneidade da vegetação, que apesar de compartilharem variáveis ambientais semelhantes (e.g. clima, precipitação, tipo de solo e geomorfologia), têm distribuição espacial em manchas, o que pode determinar uma variação estrutural causada por diferentes fatores bióticos (e.g. riqueza, diversidade e densidade da fonte de propágulos, influência das diferentes formações vegetais circunvizinhas, entre outros) (Watson, 2002; Parmentier et al., 2005).

Apesar de vários estudos apontarem para a influência do cerrado na flora dos tabuleiros, essa questão foi sempre baseada em dados pontuais. No entanto, os tabuleiros apresentam uma grande complexidade ecológica e biogeográfica (i.e. origem, diversidade e relações históricas).

Diante desse contxto, os tabuleiros costeiros apresentam uma vegetação heterogênea com variações estruturais entre diferentes manchas. Assim, o objetivo do presente estudo foi descrever e comparar quatro áreas de tabuleiro, quanto a estrutura, a riqueza e diversidade de espécies lenhosas.

\section{Material e Métodos \\ Área de estudo}

$\mathrm{O}$ estudo foi realizado em quatro áreas de tabuleiro, no estado da Paraíba: Pedras de Fogo $\left(07^{\circ} 21^{\prime} 12^{\prime \prime} \mathrm{S}, 35^{\circ} 00^{\prime} 08^{\prime \prime} \mathrm{W}\right)$, Conde (07'21'18,9'S, $\left.34^{\circ} 48^{\prime} 23,7^{\prime \prime} \mathrm{W}\right), \quad$ Mamanguape $\quad\left(06^{\circ} 40^{\prime} 42^{\prime \prime} \mathrm{S}\right.$, $\left.35^{\circ} 07^{\prime} 32^{\prime \prime} W\right)$ e João Pessoa $\left(07^{\circ} 11^{\prime} 15,3\right.$ 'S, $34^{\circ} 38^{\prime} 02,6^{\prime \prime} \mathrm{W}$ ) (Figura 1). As áreas foram escolhidas com base em informações da literatura e em viagens de reconhecimento no campo.

Todas as áreas localizam-se em terrenos de sedimentos arenosos pertencentes ao Grupo Barreiras (Ranzani et al., 1964; Mabesoone et al., 1972; Bigarella, 1975), cujo solos são classificados como Neossolos Quartzarênicos (Prates et al., 1978; EMBRAPA, 1999). A precipitação média anual varia de 1.500 a $2.000 \mathrm{~mm}^{-\mathrm{ano}^{-1}}$ e o clima, em toda a extensão, é do tipo As', segundo classificação de Köppen (Prates et al., 1981).

As áreas de Pedras de Fogo e Mamanguape são as mais distantes da linha da costa, com $19 \mathrm{~km}$ e $15 \mathrm{~km}$, respectivamente. A vegetação de tabuleiro de Pedras de Fogo está circundada por uma matriz de cana-de-açúcar, com uma distância média de 3 $\mathrm{m}$, entre a borda do plantio e a vegetação de tabuleiro. A área de Mamanguape, por sua vez, está inserida em uma Área de Proteção Ambiental, a Reserva Ecológica de Guaribas (MMA/IBAMA, 2003). Essa área está em contato com a floresta atlântica e, em determinados trechos, a área de transição forma uma pequena mata densa denominada, pelos moradores locais, de "carrasco". A vegetação de tabuleiro também tem contato com áreas de campo alagável, ocupando terrenos adjacentes mais baixos, que são inundados na época das chuvas, apresentando, em quase sua totalidade uma cobertura herbácea, com poucas 
espécies arbóreas que se destacam na paisagem, formando "moitas".

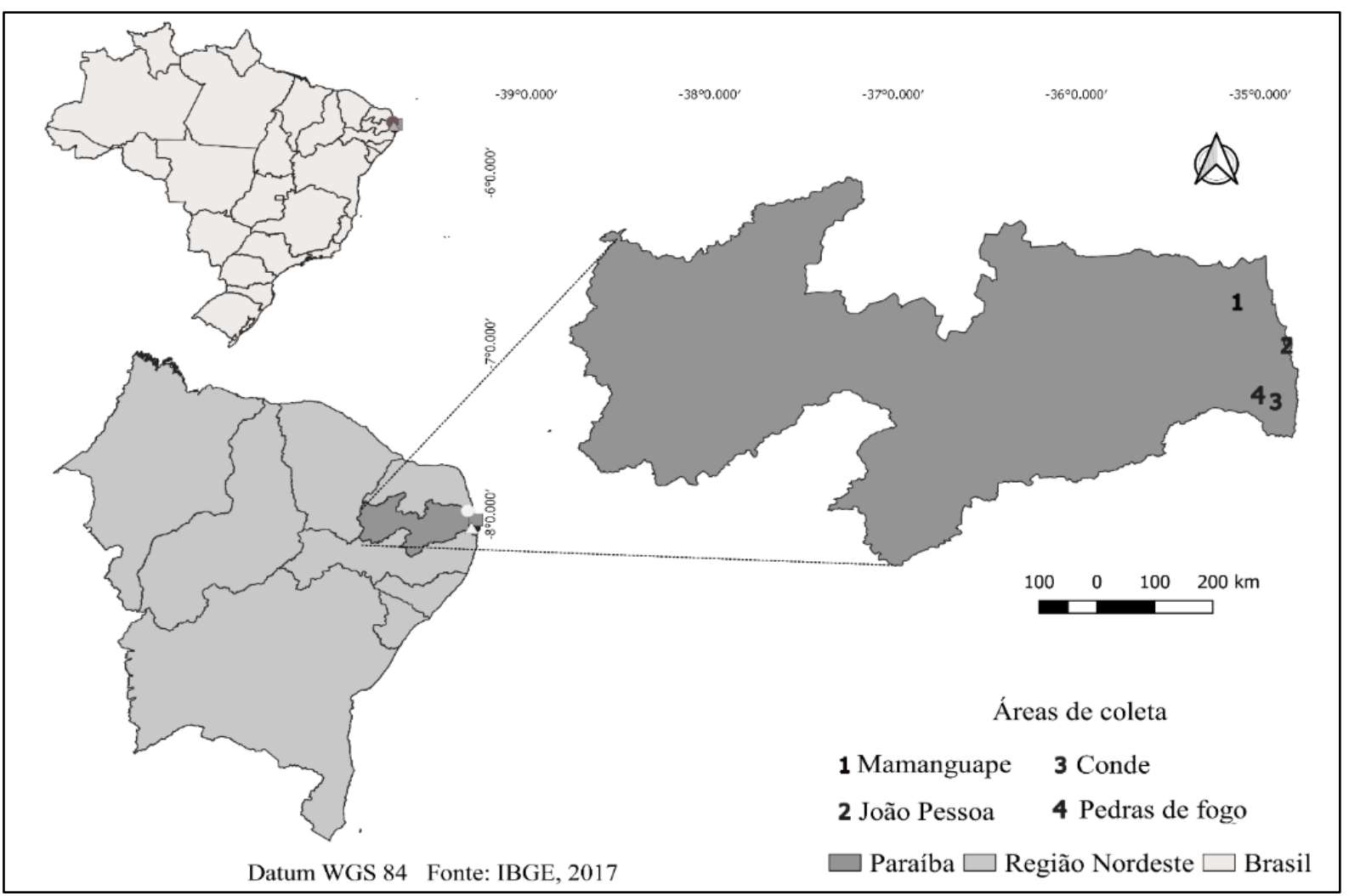

Figura 1. Localização das quatro áreas de tabuleiro costeiro (Mamanguape, João Pessoa, Conde, Pedras de Fogo) no estado da Paraíba, no Nordeste do Brasil. Fonte: Santos, C.R. (2020).

As áreas de João Pessoa e Conde estão mais próximas do litoral, e ambas ocupam terrenos planos, cujas bordas formam falésias. A área de João Pessoa é próxima à praia de Cabo Branco, inserida no perímetro urbano, sendo forte alvo de pressão antrópica. A área de Conde, situada na porção oriental do estado da Paraíba, apresenta uma vegetação alterada, devido a vários processos de ocupação do solo e retirada da vegetação pela ocupação urbana (Neves, 1993).

Estrutura, riqueza e diversidade de espécies lenhosas

A análise estrutural da vegetação foi realizada utilizando o método de pontos quadrantes (Cottam \& Curtis, 1956). Para isso foram demarcadas cinco transecções paralelas, espaçadas a $10 \mathrm{~m}$; em cada transecção foram alocados 10 pontos quadrantes, e cada ponto era espaçado a intervalos de $10 \mathrm{~m}$, totalizando 50 pontos de amostragem. A amostragem do presente estudo seguiu Almeida Jr. (2006), no qual, em áreas de restinga, os 50 pontos quadrantes são o suficiente para alcançar uma curva estável de acumulação de espécies.

Este método foi aplicado em cada área de estudo. Para amostrar os indivíduos lenhosos foi utilizado, como critério de inclusão, o perímetro na altura do solo (PAS) $>10 \mathrm{~cm}$. Os indivíduos com perfilhos foram considerados na amostragem quando o perímetro de um dos perfilhos apresentasse o valor mínimo de inclusão. Todos os indivíduos tiveram perímetro mensurado e altura estimada.

O material botânico foi coletado para posterior identificação, seguindo a proposta do APG IV (2016). O material coletado foi depositado no Herbário IPA Dárdano de Andrade Lima (Instituto Agronômico de Pernambuco), com duplicatas no Herbário Professor Vasconcelos Sobrinho (PEUFR) da Universidade Federal Rural de Pernambuco-UFRPE. Os táxons que não foram reconhecidos, quanto à família, foram considerados como "não identificados".

A partir dos dados coletados, foram calculadas densidade e área basal total (AB), dominância relativa (DoR) e densidade relativa (DR), com base nos parâmetros descritos por Martins (1991), por meio do software FITOPAC 2.1 (Shepherd, 2009). Foram calculados riqueza, diversidade, altura e diâmetro médios e analisada a proporção de indivíduos com perfilho. A diversidade e a equitabilidade foram calculadas por meio dos índices de Shannon (H') e Pielou (J'), 
respectivamente (Zar, 1999; Ayres et al., 2002). Os indivíduos foram distribuídos em classes de altura $(2 \mathrm{~m})$ e diâmetro $(3 \mathrm{~cm})$. Essa distribuição deve-se aos valores associados as plantas desse ecossistema, por possuírem troncos finos e altura mediana. Além disso, visando padronizar os valores, em relação aos estudos desenvolvidos anteriormente, foram realizadas comparações entre as áreas, por apresentarem parâmetros metodológicos semelhantes. A similaridade florística das áreas foi comparada por meio do índice de similaridade de Jaccard (Krebs 1989).

\section{Análise estatística}

A normalidade dos dados foi testada utilizando o teste de Kolmogorov-Smirnov (Zar, 1999) e as diferenças entre a altura e o diâmetro médios da vegetação das áreas de tabuleiro costeiro foram analisadas através de análise de variância (ANOVA), seguido do teste de Tukey (Zar, 1999). Para a comparação de densidade total, riqueza e proporção de indivíduos com perfilhos foi utilizado o teste $\mathrm{G}$ para as frequências de indivíduos por área (Zar, 1999). A diversidade foi comparada, par a par, por meio do teste "t" de Hutcheson (Zar, 1999). Além da comparação entre as áreas de tabuleiro costeiro do presente estudo, também foram realizadas análises com áreas de tabuleiro da região Nordeste (Zickel et al., 2012).

\section{Resultados}

Estrutura, riqueza e diversidade de espécies lenhosas

A densidade de espécies lenhosas variou de 2.838 ind.ha $^{-1}$ (Mamanguape) a 5.761 ind.ha $^{-1}$ (Pedras de Fogo), todas as áreas apresentaram diferenças significativas entre si (Tabela 1). Pedras de Fogo e João Pessoa apresentaram os maiores valores de área basal total $\left(64,95\right.$ e $55,18 \mathrm{~m}^{2} \cdot \mathrm{ha}^{-1}$, respectivamente) e foram significativamente diferentes das outras áreas (Conde e Mamanguape) (Tabela 1).

Tabela 1. Valores para altura e diâmetro médio, densidade, área basal total, riqueza, diversidade, equitabilidade e porcentagem de indivíduos com perfilho, nas áreas de tabuleiro costeiro (Mamanguape, João Pessoa, Conde, Pedras de Fogo) da Paraíba. Fonte: Zickel, C.S. (2006).

\begin{tabular}{lcccc}
\hline Parâmetros & Mamanguape & João Pessoa & Conde & Pedras de Fogo \\
\hline Altura média & $2,44 \mathrm{a} \pm 0,11$ & $4,20 \mathrm{~b} \pm 0,11$ & $5,34 \mathrm{c} \pm 0,13$ & $4,73 \mathrm{~d} \pm 0,08$ \\
Diâmetro médio & $6,67 \mathrm{~b} \pm 0,35$ & $6,48 \mathrm{ab} \pm 0,54$ & $6,66 \mathrm{~b} \pm 0,27$ & $6,47 \mathrm{ab} \pm 0,43$ \\
Densidade (ind/ha) & $2.838 \mathrm{a}$ & $5.688 \mathrm{~b}$ & $3.428 \mathrm{c}$ & $5.761 \mathrm{~d}$ \\
Área basal total (ha) & $16,95 \mathrm{a}$ & $55,18 \mathrm{bc}$ & $24,85 \mathrm{ab}$ & $64,95 \mathrm{c}$ \\
Riqueza & $23 \mathrm{a}$ & $45 \mathrm{~b}$ & $42 \mathrm{ab}$ & $32 \mathrm{ab}$ \\
Diversidade (H') nat.ind $\left.{ }^{-1}\right)$ & $2,44 \mathrm{a}$ & $3,37 \mathrm{c}$ & $3,16 \mathrm{~d}$ & $2,46 \mathrm{a}$ \\
Equitabilidade (J') & 0,77 & 0,88 & 0,84 & 0,68 \\
$\mathrm{~N}^{\mathbf{o}}$ de ind. perfilhados (\%) & $18 \mathrm{a}$ & $32 \mathrm{bc}$ & $34 \mathrm{bc}$ & $27,5 \mathrm{ab}$ \\
\hline
\end{tabular}

Letras diferentes após o número indicam diferença significativa entre médias comparadas pelo teste a posteriori de Tukey, teste $\mathrm{t}$ de Hutcheson e entre frequências pelo teste $\mathrm{G}$, analisadas par a par.

A altura média dos indivíduos variou de 2,44 m em Mamanguape, a 5,34 $\mathrm{m}$ em Conde (Tabela 1). A média dos diâmetros variou de 6,47 $\mathrm{cm}$ a 6,67 cm, entre as áreas de Pedras de Fogo e Mamanguape (Tabela 1). Na análise da proporção de indivíduos com perfilhos, Mamanguape mostrou diferença significativa com relação a João Pessoa e Conde, enquanto em Pedras de Fogo os valores representaram uma variação total de $16 \%$ entre a área com maior proporção de indivíduos perfilhados, Conde (34\%), e a de menor proporção, Mamanguape (18\%) (Tabela 1).

As únicas áreas que apresentaram diferença significativa quanto ao número de espécies foram Mamanguape e João Pessoa, com 23 e 45 espécies, respectivamente (Tabela 1). Estas duas áreas também apresentaram o menor e o maior valor de diversidade $\left(H^{\prime}\right)$ 2,44 nats.ind ${ }^{-1}$ e 3,37 nats.ind ${ }^{-1}$ respectivamente (Tabela 1). Mamanguape e Pedras de Fogo não mostraram diferença significativa entre si. A equitabilidade (J') variou de 0,68, em Pedras de Fogo, a 0,88, em João Pessoa (Tabela 1).

As famílias mais ricas em espécies foram Fabaceae (15 espécies), Rubiaceae (oito espécies), Sapotaceae e Myrtaceae (com seis espécies, cada). Nas quatro áreas analisadas, poucas espécies foram responsáveis por mais da metade da dominância relativa (DoR). Em Mamanguape, João Pessoa e Conde destacaram-se quatro espécies em cada área, sendo: Hirtella ciliata Mart. \& Zucc., Ouratea fieldingiana (Gardner) Engl., Hancornia speciosa Gomes e Anacardium occidentale L., em Mamanguape; Coccoloba latifolia Lam., Himatanthus phagedaenicus (Mart.) Woodson, Protium heptaphyllum (Aubl.) Marchand e Eschweilera ovata (Cambess.) Mart. ex Miers, em João Pessoa; Tapirira guianensis Aubl., Byrsonima sericea DC., Stryphnodendron pulcherrimum (Willd.) Hochr. e Lecythis pisonis Cambess., em 
Conde. Na área de Pedras de Fogo apenas uma espécie (Chamaecrista bahiae (H.S. Irwin) H.S.
Irwin \& Barneby) representou metade da DoR total (Tabela 2).

Tabela 2. Espécies registradas nas quatro áreas de tabuleiro costeiro (Mamanguape, João Pessoa, Conde, Pedras de Fogo) da Paraíba, ordenadas a partir da maior dominância relativa (DoR). N. col = número do coletor (A. Vicente /IPA), DR = densidade relativa, $\mathrm{AB}=$ área basal. Às espécies não identificadas foram acrescentadas as iniciais do município. Fonte: Zickel, C.S. (2006).

\begin{tabular}{|c|c|c|c|c|c|}
\hline Espécies & Família & N. col. & DoR & DR & $\mathbf{A B}$ \\
\hline \multicolumn{6}{|l|}{ MAMANGUAPE } \\
\hline Hirtella ciliata Mart. \& Zucc. & Chrysobalanaceae & 244 & 22,76 & 10,5 & 0,2719 \\
\hline Ouratea fieldingiana (Gardner) Engl. & Ochnaceae & 246 & 14,05 & 22,5 & 0,1678 \\
\hline Hancornia speciosa Gomes & Apocynaceae & 268 & 12,86 & 18,0 & 0,1536 \\
\hline Anacardium occidentale L. & Anacardiaceae & 265 & 12,72 & 1,0 & 0,1519 \\
\hline Byrsonima cydoniifolia A. Juss. & Malpighiaceae & 245 & 7,91 & 8,0 & 0,0944 \\
\hline Guettarda platypoda DC. & Rubiaceae & 267 & 7,03 & 5,0 & 0,0840 \\
\hline Curatella americana L. & Dilleniaceae & 266 & 6,01 & 0,5 & 0,0718 \\
\hline Myrcia bergiana $\mathrm{O}$. Berg. & Myrtaceae & 269 & 4,70 & 5,5 & 0,0562 \\
\hline Maytenus distichophylla Mart. ex Reissek & Celastraceae & 250 & 3,55 & 11,5 & 0,0424 \\
\hline Campomanesia dichotoma (O. Berg.) Mattos & Myrtaceae & 252 & 2,31 & 2,0 & 0,0276 \\
\hline Miconia albicans $(\mathrm{Sw})$ Triana & Melastomataceae & 255 & 1,49 & 4,0 & 0,0178 \\
\hline Allophylus edulis (A.St.-Hil. et al.) Hieron. ex Niederl. & Sapindaceae & 260 & 1,16 & 2,0 & 0,0139 \\
\hline Pera glabrata (Schott.) Poepp. ex Baill. & Peraceae & 249 & 1,00 & 1,5 & 0,0119 \\
\hline Abarema filamentosa (Benth.) Pittier & Fabaceae & 247 & 0,67 & 2,0 & 0,0080 \\
\hline Coccoloba cordifolia Meisn. & Polygonaceae & 259 & 0,40 & 1,5 & 0,0047 \\
\hline Tetracera breyniana Schltdl. & Dilleniaceae & 254 & 0,28 & 0,5 & 0,0033 \\
\hline N/identificada $1 \mathrm{M}$ & - & 270 & 0,27 & 0,5 & 0,0032 \\
\hline N/identificada $2 \mathrm{M}$ & - & 271 & 0,22 & 0,5 & 0,0026 \\
\hline Salzmannia nitida DC. & Rubiaceae & 257 & 0,19 & 1,0 & 0,0023 \\
\hline Byrsonima gardneriana A. Juss. & Malpighiaceae & 264 & 0,19 & 0,5 & 0,0023 \\
\hline Thyrsodium spruceanum Benth. & Anacardiaceae & 273 & 0,08 & 0,5 & 0,0010 \\
\hline Schoepfia brasiliensis A.DC. & Schoepfiaceae & 272 & 0,08 & 0,5 & 0,0010 \\
\hline Erythroxylum aff. rimosum O.E.Schulz & Erythroxylaceae & 263 & 0,06 & 0,5 & 0,0007 \\
\hline
\end{tabular}

\section{JOÃO PESSOA}

Coccoloba latifolia Lam.
Himatanthus phagedaenicus (Mart.) Woodson
Protium heptaphyllum (Aubl.) Marchand
Eschweilera ovata (Cambess.) Mart. ex Miers
Pouteria gardneri (Mart. \& Miq.) Baehni
Annona pickelii (Diels) H.Rainer
Pradosia sp.
Inga capitata Desv.
Licania octandra (Hoffmanns. ex Roem. \& Schult.) Kuntze
Tapirira guianensis Aubl.
Lecythis pisonis Cambess.
Bowdichia virgilioides Kunth
Ocotea cf. canaliculata (Rich.) Mez
Thyrsodium spruceanum Benth.
Ocotea duckei Vattimo-Gil
Talisia sp.
Pera glabrata (Schott) Poepp. ex Baill.
Cupania racemosa (Vell.) Radlk.
Sacoglottis mattogrossensis Malme
Psychotria bahiensis DC.
N/identificada 1 JP
Myrcia sp.
Maytenus distichophylla Mart. ex Reissek
Guettarda viburnoides Cham. \& Schltdl.
Byrsonima sericea DC.
N/identificada $5 \mathrm{JP}$
Guettarda platypoda DC.
Pogonophora schomburgkiana Miers ex Benth.

\begin{tabular}{|l|l} 
Polygonaceae & 17 \\
Apocynaceae & 23 \\
Burseraceae & 172 \\
Lecythidaceae & 183 \\
Sapotaceae & 200 \\
Annonaceae & 194 \\
Sapotaceae & 178 \\
Fabaceae & 17 \\
Chrysobalanaceae & 18 \\
Anacardiaceae & 235 \\
Lecythidaceae & 190 \\
Fabaceae & 203 \\
Lauraceae & 235 \\
Anacardiaceae & 236 \\
Lauraceae & 198 \\
Sapindaceae & 237 \\
Peraceae & 238 \\
Sapindaceae & 188 \\
Humiriaceae & 139 \\
Rubiaceae & 187 \\
- & 239 \\
Myrtaceae & 168 \\
Celastraceae & 197 \\
Rubiaceae & 186 \\
Malpighiaceae & 240 \\
- & 24 \\
Rubiaceae & 242 \\
Peraceae & 202 \\
&
\end{tabular}

$\begin{array}{cccc}171 & 28,04 & 4,5 & 0,5441 \\ 234 & 14,11 & 2,5 & 0,2738 \\ 172 & 7,35 & 4,0 & 0,1426 \\ 183 & 7,10 & 6,0 & 0,1377 \\ 200 & 4,6 & 6,0 & 0,0893 \\ 194 & 4,47 & 9,5 & 0,0867 \\ 178 & 4,47 & 2,0 & 0,0867 \\ 170 & 3,97 & 6,0 & 0,0771 \\ 181 & 3,02 & 9,5 & 0,0586 \\ 235 & 2,45 & 1,0 & 0,0476 \\ 190 & 2,27 & 5,5 & 0,0440 \\ 203 & 1,95 & 0,5 & 0,0379 \\ 235 & 1,82 & 1,0 & 0,0353 \\ 236 & 1,45 & 5,5 & 0,0280 \\ 198 & 1,39 & 1,5 & 0,0269 \\ 237 & 0,99 & 4,0 & 0,0192 \\ 238 & 0,98 & 1,0 & 0,0189 \\ 188 & 0,89 & 2,5 & 0,0173 \\ 139 & 0,73 & 2,0 & 0,0142 \\ 187 & 0,66 & 0,5 & 0,0127 \\ 239 & 0,66 & 0,5 & 0,0127 \\ 168 & 0,63 & 0,5 & 0,0122 \\ 197 & 0,59 & 2,0 & 0,0114 \\ 186 & 0,59 & 3,0 & 0,0114 \\ 240 & 0,55 & 2,5 & 0,0107 \\ 241 & 0,5 & 2,0 & 0,0097 \\ 242 & 0,46 & 1,0 & 0,0090 \\ 202 & 0,38 & 0,5 & 0,0074\end{array}$


Zollernia ilicifolia (Brongn.) Vogel

Cordia superba Cham.

N/identificada 4JP

N/identificada 2JP

Myrcia guianensis (Aubl.) DC.

N/identificada 3JP

Abarema filamentosa (Benth.) Pittier

Chamaecrista ensiformis (Vell.) H.S. Irwin \& Barneby

Vismia guianensis (Aubl.) Choisy

Protium bahianum Daly

Annonaceae 1

Andira legalis (Vell.) Toledo

Guapira laxa (Netto) Furlan

Myrcia bergiana $\mathrm{O}$. Berg.

Buchenavia tetraphylla (Aubl.) R.A.Howard

Cordiera sessilis (Vell.) Kuntze

\begin{tabular}{|l|l} 
Fabaceae & 199 \\
Boraginaceae & 243 \\
- & 212 \\
- & 210 \\
Myrtaceae & 204 \\
- & 211 \\
Fabaceae & 189 \\
Fabaceae & 193 \\
Hypericaceae & 216 \\
Burseraceae & 215 \\
Annonaceae & 206 \\
Fabaceae & 213 \\
Nyctaginaceae & 201 \\
Myrtaceae & 197 \\
Combretaceae & 207 \\
Rubiaceae & 214
\end{tabular}

0,37

0,35

0,29

0,22

0,21

0,19

0,19

0,18

0,17

0,16

0,15

0,13

0,12

0,09

0,05

$0,04 \quad 0,5$

1,0

1,0

0,0072

1,0

0,0067

0,5

0,5

0,5

1,5

1,0

1,5

1,0

0,5

0,5

0,5

0,5

0,5
0,0056

0,0043

0,0041

0,0036

0,0037

0,0034

0,0033

0,0032

0,0029

0,0025

0,0023

0,0018

0,0010

0,0008

\section{CONDE}

Tapirira guianensis Aubl.

Byrsonima sericea DC.

Stryphnodendron pulcherrimum (Willd.) Hochr.

Lecythis pisonis Cambess.

Apuleia leiocarpa (Vogel) J.F. Macbr.

Guettarda viburnoides Cham. \& Schltdl.

Coccoloba mollis Casar.

Sacoglottis mattogrossensis Malme

Eschweilera ovata (Cambess.) Mart. ex Miers

Pera glabrata (Schott.) Poepp. ex Baill.

Abarema filamentosa (Benth.) Pittier

Pouteria gardneri (Mart. \& Miq.) Baehni

Chamaecrista ensiformis (Vell.) H.S. Irwin \& Barneby

$\mathrm{N} /$ identificada 2C

Inga capitata Desv.

Ocotea duckei Vattimo-Gil

Himatanthus phagedaenicus (Mart.) Woodson

Protium heptaphyllum (Aubl.) Marchand

Luehea aff. paniculata Mart. \& Zucc.

Thyrsodium spruceanum Benth.

Cupania impressinervia Acev.-Rodr.

Coccoloba cf. cordifolia Meisn.

Pouteria sp.

Licania octandra (Hoffmanns. ex Roem. \& Schult.) Kuntze Myrcia bergiana $\mathrm{O}$. Berg.

Cordia superba Cham.

Annona sp.

Cupania racemosa (Vell.) Radlk.

Cupania sp.

Palicourea sp.

Myrcia cf. splendens (Sw.) DC.

Allophylus edulis (A.St.-Hil. et al.) Hieron. ex Niederl.

Inga aff. laurina (Sw.) Willd.

Hymenaea courbaril L.

Sapotaceae 1

Manilkara salzmannii (A.DC.) H.J.Lam

N/identificada 1C

Annona pickelii (Diels) H.Rainer

Dialium sp.

Guapira pernambucensis (Casar.) Lundell

Zollernia ilicifolia (Brongn.) Vogel

Anaxagorea dolichocarpa Sprague \& Sandwith
Anacardiaceae

Malpighiaceae

Fabaceae

Lecythidaceae

Fabaceae

Rubiaceae

Polygonaceae

Humiriaceae

Lecythidaceae

Peraceae

Fabaceae

Sapotaceae

Fabaceae

-

Fabaceae

Lauraceae

Apocynaceae

Burseraceae

Malvaceae

Anacardiaceae

Sapindaceae

Polygonaceae

Sapotaceae

Chrysobalanaceae

Myrtaceae

Boraginaceae

Annonaceae

Sapindaceae

Sapindaceae

Rubiaceae

Myrtaceae

Sapindaceae

Fabaceae

Fabaceae

Sapotaceae

Sapotaceae

-

Annonaceae

Fabaceae

Nyctaginaceae

Fabaceae

Annonaceae

$\begin{array}{cccc}71 & 29,96 & 15,0 & 0,4344 \\ 64 & 9,57 & 12,0 & 0,1387 \\ 80 & 7,63 & 4,0 & 0,1107 \\ 59 & 6,17 & 8,0 & 0,0895 \\ 115 & 5,82 & 2,0 & 0,0843 \\ 120 & 3,57 & 7,0 & 0,0518 \\ 101 & 3,46 & 3,5 & 0,0501 \\ 109 & 3,33 & 1,5 & 0,0483 \\ 95 & 3,32 & 4,5 & 0,0482 \\ 100 & 2,66 & 1,5 & 0,0385 \\ 66 & 2,43 & 2,5 & 0,0352 \\ 70 & 2,39 & 3,0 & 0,0346 \\ 60 & 2,17 & 3,0 & 0,0314 \\ 125 & 2,10 & 0,5 & 0,0305 \\ 122 & 2,00 & 4,0 & 0,0290 \\ 86 & 1,61 & 4,5 & 0,0233 \\ 88 & 1,31 & 3,0 & 0,0189 \\ 110 & 1,20 & 0,5 & 0,0174 \\ 94 & 1,19 & 1,0 & 0,0172 \\ 76 & 0,89 & 3,0 & 0,0129 \\ 124 & 0,86 & 0,5 & 0,0125 \\ 116 & 0,79 & 1,5 & 0,0115 \\ 130 & 0,60 & 0,5 & 0,0087 \\ 103 & 0,59 & 1,0 & 0,0086 \\ 93 & 0,56 & 0,5 & 0,0082 \\ 117 & 0,55 & 1,5 & 0,0080 \\ 62 & 0,48 & 0,5 & 0,0070 \\ 84 & 0,40 & 0,5 & 0,0058 \\ 118 & 0,40 & 0,5 & 0,0058 \\ 107 & 0,30 & 0,5 & 0,0043 \\ 69 & 0,25 & 1,5 & 0,0037 \\ 73 & 0,24 & 2,0 & 0,0035 \\ 81 & 0,21 & 0,5 & 0,0030 \\ 121 & 0,20 & 0,5 & 0,0029 \\ 129 & 0,15 & 0,5 & 0,0022 \\ 65 & 0,14 & 0,5 & 0,002 \\ 123 & 0,12 & 0,5 & 0,0018 \\ 110 & 0,09 & 0,5 & 0,0013 \\ 127 & 0,08 & 0,5 & 0,0012 \\ 120 & 0,08 & 0,5 & 0,0011 \\ 126 & 0,07 & 0,5 & 0,0010 \\ 114 & 0,05 & 0,5 & 0,0008\end{array}$


Stryphnodendron pulcherrimum (Willd.) Hochr.

Anadenanthera colubrina (Vell.) Brenan

Coccoloba ramosissima Wedd.

$\mathrm{N} /$ identificada $2 \mathrm{PF}$

$\mathrm{N} /$ identificada $1 \mathrm{PF}$

Buchenavia tetraphylla (Aubl.) R.A.Howard

Andira nitida Mart. ex Benth.

Esenbeckia grandiflora Mart.

Cecropia pachystachya Trécul

Guettarda platypoda DC.

Coccoloba mollis Casar.

Byrsonima sericea DC.

Inga sp.

Ocotea gardneri (Meisn.) Mez

Licania octandra (Hoffmanns. ex Roem. \& Schult.) Kuntze

Sacoglottis mattogrossensis Malme

Eriotheca macrophylla (K.Schum.) A.Robyns

Thyrsodium spruceanum Benth.

Duguetia lanceolata A. St.-Hil.

Guapira laxa (Netto) Furlan

Pouteria gardneri (Mart. \& Miq.) Baehni

Inga capitata Desv.

Chaetocarpus myrsinites Baill.

Pouteria sp.

Cupania racemosa (Vell.) Radlk.

Pouteria gardneri (Mart. \& Miq.) Baehni

Myrcia sp.

Psychotria hoffmannseggiana (Willd. ex Schult.) Müll.Arg. Chamaecrista ensiformis (Vell.) H.S. Irwin \& Barneby

Clusia dardanoi G. Mariz \& Maguire

Maytenus distichophylla Mart. ex Reissek

\begin{tabular}{|c|c|c|c|c|}
\hline Fabaceae & 348 & 15,23 & 3,5 & 0,3434 \\
\hline Fabaceae & 313 & 14,73 & 6,5 & 0,3320 \\
\hline Polygonaceae & 320 & 2,07 & 6,5 & 0,0466 \\
\hline- & 341 & 1,55 & 0,5 & 0,035 \\
\hline- & 340 & 1,31 & 5,5 & 0,0295 \\
\hline Combretaceae & 332 & 1,31 & 1,0 & 0,0296 \\
\hline Fabaceae & 331 & 0,95 & 2,0 & 0,0215 \\
\hline Rutaceae & 336 & 0,86 & 3,5 & 0,0194 \\
\hline Urticaceae & 334 & 0,77 & 2,0 & 0,0173 \\
\hline Rubiaceae & 337 & 0,56 & 0,5 & 0,0127 \\
\hline Polygonaceae & 311 & 0,55 & 3,5 & 0,0124 \\
\hline Malpighiaceae & 333 & 0,47 & 1,0 & 0,0106 \\
\hline Fabaceae & 343 & 0,46 & 0,5 & 0,0103 \\
\hline Lauraceae & 325 & 0,43 & 1,0 & 0,0097 \\
\hline Chrysobalanaceae & 323 & 0,43 & 0,5 & 0,0097 \\
\hline Humiriaceae & 312 & 0,36 & 0,5 & 0,0082 \\
\hline Malvaceae & 335 & 0,30 & 0,5 & 0,0067 \\
\hline Anacardiaceae & 318 & 0,29 & 1,5 & 0,0065 \\
\hline Annonaceae & 328 & 0,29 & 1,0 & 0,0066 \\
\hline Nyctaginaceae & 324 & 0,24 & 0,5 & 0,0054 \\
\hline Sapotaceae & 327 & 0,21 & 1,5 & 0,0047 \\
\hline Fabaceae & 342 & 0,20 & 1,0 & 0,0045 \\
\hline Peraceae & 329 & 0,19 & 1,0 & 0,0043 \\
\hline Sapotaceae & 347 & 0,16 & 0,5 & 0,0035 \\
\hline Sapindaceae & 345 & 0,08 & 1,0 & 0,0018 \\
\hline Sapotaceae & 346 & 0,06 & 0,5 & 0,0013 \\
\hline Myrtaceae & 338 & 0,06 & 0,5 & 0,0014 \\
\hline Rubiaceae & 330 & 0,05 & 0,5 & 0,0012 \\
\hline Fabaceae & 344 & 0,04 & 0,5 & 0,0010 \\
\hline Clusiaceae & 339 & 0,04 & 0,5 & 0,0008 \\
\hline Celastraceae & 317 & 0,04 & 0,5 & 0,0008 \\
\hline
\end{tabular}

As áreas que apresentaram maior semelhança florística foram João Pessoa e Conde com $42 \%$ de similaridade. As áreas que não apresentaram similaridade foram Mamanguape e Pedras de Fogo (8\%) (Tabela 3).

Tabela 3. Relação de similaridade (\%) entre as quatro áreas de tabuleiro costeiro (Mamanguape, João Pessoa, Conde, Pedras de Fogo) da Paraíba, calculados através do índice de similaridade de Jaccard. Fonte: Zickel, C.S. (2006).

\begin{tabular}{lccc}
\hline Locais & Mamanguape & $\begin{array}{c}\text { João } \\
\text { Pessoa }\end{array}$ & Conde \\
\hline João & 15 & & \\
Pessoa & 11 & 42 & \\
Conde & 8 & 21 & 14 \\
Pedras de & Fogo & &
\end{tabular}

Distribuição de indivíduos em classes de altura e diâmetro

As áreas estudadas apresentaram padrões de distribuição de altura diferentes entre si. A distribuição de frequências de altura das árvores (Figura 2) indicou que João Pessoa foi a área com maior concentração de indivíduos na segunda classe, variando de $2-4 \mathrm{~m}(53 \%)$ e na terceira classe, variando de 4-6 m (29,5\%). Mamanguape teve um maior percentual de indivíduos na classe de $0-2 \mathrm{~m}(59,5 \%)$ e distribuição decrescente em direção as classes de maior altura. A área de Conde mostrou a mesma porcentagem de indivíduos nas classes de 2-4 m e 4-6 m (33\%). Pedras de Fogo apresentou uma distribuição descontínua, com poucos indivíduos nas classes de 0-2 m (1\%) e 4-6 $\mathrm{m}(0,5 \%)$, e maior concentração nas classes de 6-8 $\mathrm{m}(48,5 \%)$ e $2-4 \mathrm{~m}(42 \%)$.

A variação na distribuição das diferenças diamétricas foi muito semelhante entre as áreas (Figura 3), principalmente entre as áreas de Mamanguape, João Pessoa e Pedras de Fogo, apresentando uma maior concentração de indivíduos na classe de 3-6 cm e na classe de 6-9 $\mathrm{cm}$. A área de Conde apresentou uma distribuição de indivíduos mais proporcional entre as classes, com cerca de $50 \%$ na classe de $3-6 \mathrm{~cm}$ e $30 \%$ na classe de 6-9 $\mathrm{cm}$. 


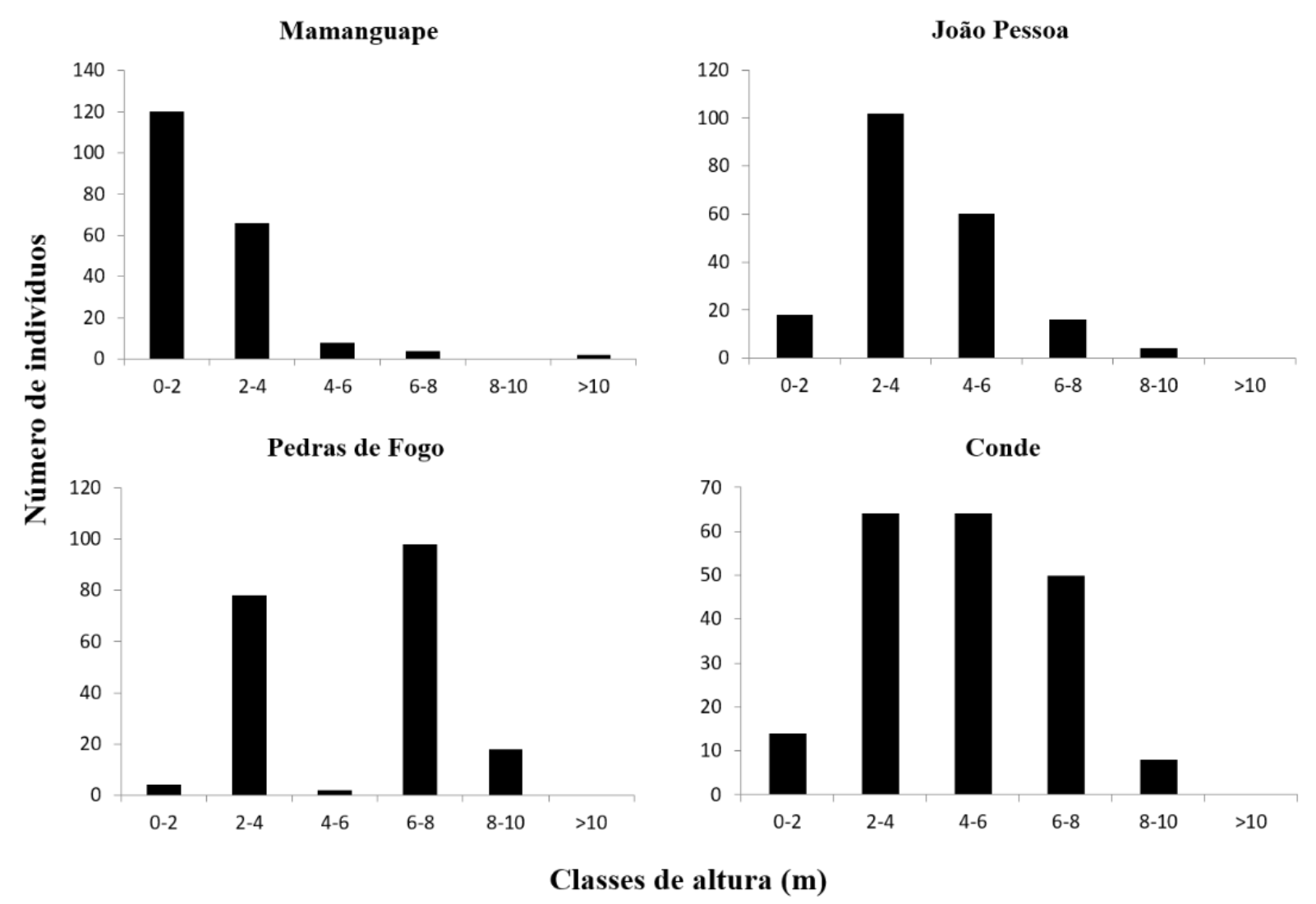

Figura 2. Distribuição de indivíduos por classe de altura nas quatro áreas de tabuleiro costeiro (Mamanguape, João Pessoa, Conde, Pedras de Fogo) da Paraíba. Fonte: Zickel et al. (2020).
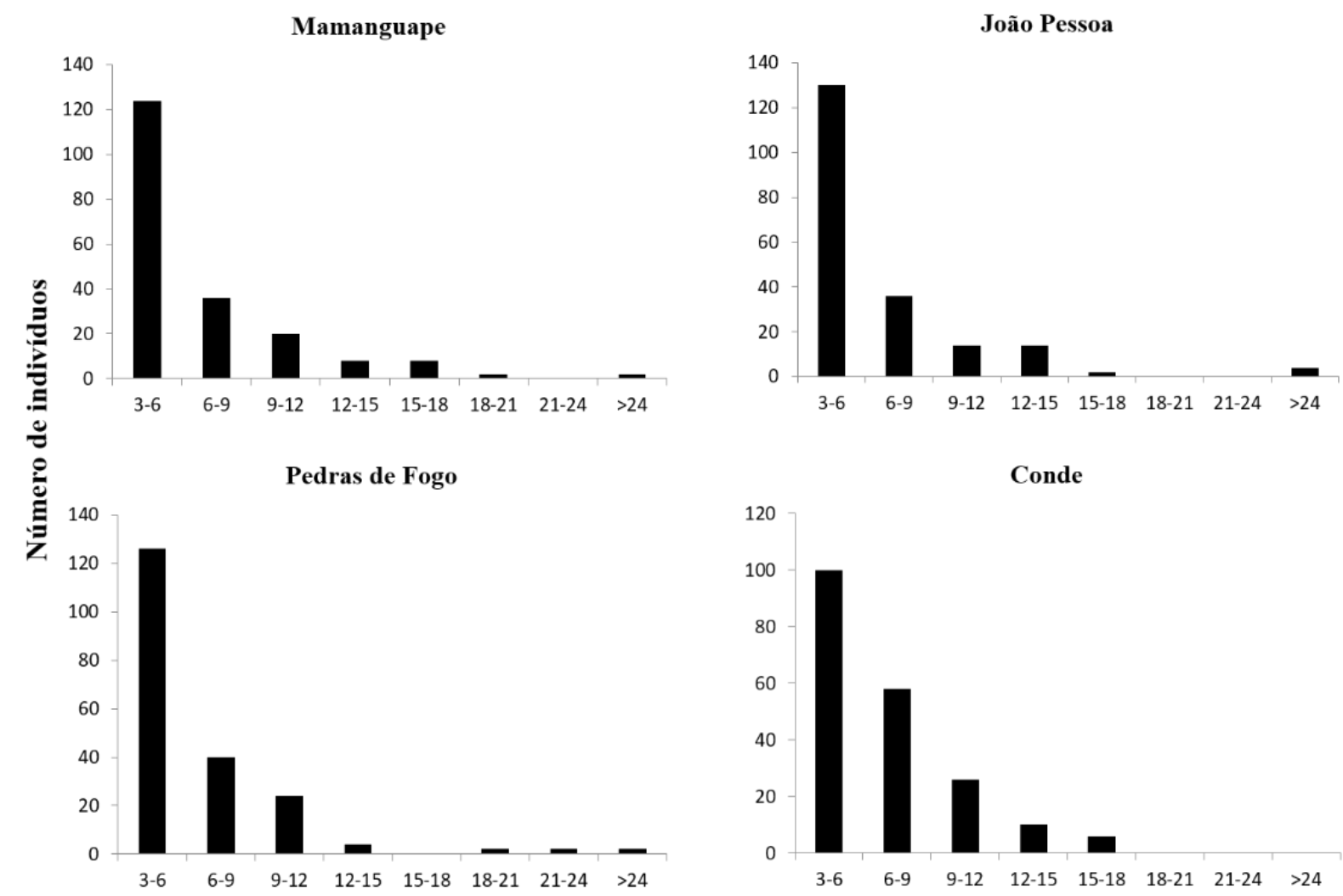

Classes de diâmetros $(\mathrm{cm})$

Figura 3. Distribuição de indivíduos por classe de diâmetro nas quatro áreas de tabuleiro costeiro (Mamanguape, João Pessoa, Conde, Pedras de Fogo) da Paraíba. Fonte: Zickel et al. (2020). 


\section{Discussão}

As áreas analisadas no presente estudo apresentaram valores diferentes de densidade, área basal, diversidade, média das alturas e diâmetros, proporção de indivíduos com perfilhos e composição da flora. Estes resultados indicam que a heterogeneidade das comunidades vegetais pode estar associada aos fatores climáticos, edáficos e topográficos e que podem explicar as fitofisionomias distintas, observadas ao longo dos tabuleiros (Tavares, 1960; 1964; Andrade-Lima, 1960; 1970; Oliveira-Filho, 1993).

Os tabuleiros costeiros podem ser considerados como ilhas de vegetação aberta, que apresentam composição similar ao cerrado e a floresta atlântica (Vicente, 2006). Todavia, devido à fragmentação das grandes florestas, essas áreas não mantêm contato direto, salvo a área de Mamanguape, onde a floresta existente ainda envolve a área de tabuleiro. Essa caracterização é importante para se entender a variação entre as áreas estudadas. Os fragmentos remanescentes são distintos por possuírem contato com outras manchas de vegetação, enquanto a diversidade dessas áreas pode ser proveniente das espécies colonizadoras advindas de fragmentos vizinhos (Watson, 2002).

$\mathrm{O}$ arranjo estrutural desses fragmentos de vegetação de tabuleiro sugere que as variações encontradas estejam diretamente relacionadas ao componente dominante que podem variar de acordo com as assembleias de espécies (Dias et al., 2005). Entre os componentes, considerando os fatores locais e regionais (Akatov et al., 2005;), destacam-se a restrição ambiental quanto aos nutrientes do solo, disponibilidade de água, profundidade do lençol freático, nível de antropização, por exemplo. Quando mudamos a escala para a regional, nichos ecologicamente correspondentes podem ser ocupados por diferentes espécies, dependendo do conjunto de espécies localmente viáveis.

Os valores de diversidade (H') variaram de 2,44 nats.ind ${ }^{-1}$ a 3,37 nats.ind ${ }^{-1}$ (Tabela 1). Os valores de diversidade encontrados em Mamanguape podem estar associados à fitofisionomia da área, que devido às inundações ocorrentes possibilita o desenvolvimento de mais espécies herbáceas, reduzindo o número de plantas lenhosas no ambiente. $\mathrm{O}$ índice de diversidade de 3,37 encontrado em João Pessoa se aproxima ao valor encontrado na Mata do Buraquinho $\left(\mathrm{H}^{\prime}=\right.$ 3,05), remanescente da floresta Atlântica na Paraíba (Barbosa, 1996). Porém, este valor é considerado baixo quando comparado com o estudo de Magalhães (2018), que obteve um H' de 4,75 para os tabuleiros costeiros na Bahia, em uma floresta em bom estado de conservação. Isto pode ser justificado pela alta diversidade registrada em tabuleiros ao sul da Bahia (Carvalho, 2000), além das pressões antrópicas presentes em João Pessoa.

Os valores fitossociológicos encontrados nesse estudo também são observados em outros estudos quantitativos realizados no litoral do Nordeste brasileiro e que adotaram metodologias semelhantes. Como o estudo da vegetação de tabuleiro costeiros em Macaíba, realizado no Rio Grande do Norte por Cestaro \& Soares (2004) registraram uma densidade de 1.924 ind.ha $^{-1}, 40$ espécies e diversidade de 3,26 nats.ind ${ }^{-1}$.

De maneira geral, os tabuleiros situados mais ao sul da área de estudo apresentaram maiores valores para a maioria dos parâmetros analisados (riqueza, densidade total, altura média, diâmetro médio e área basal total). Este fato também deve estar associado à influência das áreas circunvizinhas, pois estes tabuleiros estão em contato com áreas que originalmente eram ocupadas pela floresta ombrófila densa e ombrófila aberta (IBGE, 1985).

Carvalho (2010) também encontrou alta riqueza das famílias Fabaceae, Rubiaceae e Sapotaceae nas comunidades vegetais de tabuleiros na Bahia. As famílias que apresentaram maior número de espécies são predominantes em estudos florísticos e fitossociológicos para a floresta Atlântica, indicando a influência do bioma na diversidade da flora dos tabuleiros (Oliveira-Filho $\&$ Fontes, 2000).

As espécies que apresentaram maior dominância relativa, em cada área estudada, parecem refletir o padrão de colonização desses tabuleiros. Por exemplo, em João Pessoa, Conde e Pedras de Fogo, as espécies dominantes como Coccoloba latifolia, Himatanthus phagedaenicus, Protium heptaphyllum, Eschweilera ovata, Tapirira guianensis, Byrsonima sericea, Lecythis pisonis e Chamaecrista bahiae são abundantes nas florestas Ombrófilas Costeiras do Nordeste (Barbosa, 1996; Guedes, 1998). Estas áreas também apresentaram um maior conjunto de espécies em comum, o que reforça a ideia da origem, a partir da mesma matriz.

Por outro lado, a área mais ao norte, Mamanguape, apresentou espécies dominantes que são mais abundantes na restinga, como Ouratea fieldingiana, Hancornia speciosa, Anacardium occidentale, Chamaecrista ensiformis e Byrsonima verbascifolia (Almeida Jr. et al., 2009; Cantarelli et al., 2012; Zickel et al., 2015), e na caatinga, como Piptadenia moniliformis. Dados que corroboram Zickel et al. (2012) no estudo de duas áreas de tabuleiro costeiros do Rio Grande do Norte. A espécie Piptadenia moniliformis é abundante em 
áreas de solo arenoso desde o Maranhão até a Bahia, principalmente na caatinga (Salgado et al., 1981, Cestaro \& Soares, 2004) e apresentou maiores densidade e dominância relativa em Parnamirim, no Rio Grande do Norte (Zickel et al., 2012).

A riqueza, a diversidade e a estrutura espacial da vegetação resultam de múltiplas causas, com diferentes fatores interagindo $\mathrm{e}$, frequentemente, resultando em um efeito de sobreposição no espaço (Parmentier et al., 2005). Esse fato pode justificar a distribuição de espécies estar relacionada com a heterogeneidade de hábitats (Condit et al., 2002; Phillips et al., 2003; Ivanauskas et al., 2004). Muitas espécies tropicais não estão distribuídas aleatoriamente em relação às variáveis ambientais, e seus padrões refletem hábitats preferenciais ou variações na história de vida dessas espécies. Possivelmente, espécies arbóreas com baixa densidade são sensíveis à heterogeneidade ambiental e as poucas espécies dominantes são relativamente indiferentes a essa heterogeneidade (Pitman et al., 2001). Embora as espécies raras contribuam, significativamente, para a riqueza, a maior ênfase deve ser direcionada à pequena proporção de espécies que ocorrem com alta frequência e densidade local, formando oligarquias previsíveis (Pitman et al., 2001).

Oligarquia é uma característica comum em hábitats sujeitos a condições ambientais extremas e o funcionamento dessas comunidades conta com essas poucas espécies (Scarano, 2002; Cirne et al., 2003; Dias et al., 2005). Dias et al. (2005), estudando 30 manchas de restinga no sudeste do Brasil, com condições ambientais semelhantes, demonstraram que áreas com diferentes espécies dominantes apresentaram diferenças na estrutura da vegetação. Estudos realizados em tabuleiros costeiros e restingas do nordeste do Brasil demonstram que poucas espécies (entre 4 e 5) representam mais de $50 \%$ da dominância relativa total (Cestaro \& Soares, 2004). Os dados do presente estudo concordam com esse fato, apresentando entre uma a quatro espécies com maior dominância.

A estratificação vertical é o reflexo da distribuição dos indivíduos dentro do estrato, tais dados podem ser utilizados para mensurar efeitos de distúrbio na vegetação, como pode ser exemplificado pela área de Pedras de Fogo que apresenta uma distribuição descontinuada em relação às classes de altura, podendo ser um indicativo de que algum fator abiótico pode estar afetando o crescimento dos indivíduos na comunidade (Roth, 1999; Hitimana et al., 2004), como a retirada seletiva de madeira ou a derrubada predatória, por exemplo.
No Rio Grande do Norte, nas áreas de Nísia Floresta e Parnamirim, o padrão de distribuição de frequências de diâmetro apresenta a maior porcentagem de indivíduos na primeira classe $(\geq 75 \%)$, refletindo o tipo de pressão exercida sobre essas áreas, que, por estarem próximas de um grande centro urbano, estão sujeitas a exploração da vegetação para diversos fins (Zickel et al., 2012).

Vegetações abertas trazem, como característica, o alto número de indivíduos perfilhados, mas esse fator pode ser intensificado por distúrbios causados por práticas humanas (Dunphy et al., 2000; Weaver \& Chinea, 2003). A proporção de indivíduos perfilhados reforça essa suposição para áreas de Nísia Floresta e Parnamirim, que apresentaram taxas de indivíduos com perfilhos com $43 \%$ e $44,5 \%$, respectivamente (Zickel et al., 2012). Na área de Mamanguape, que se apresenta como mais conservada, foi registrada uma baixa proporção de indivíduos com perfilhos (18\%). Oliveira (2002) encontrou uma diferença de, aproximadamente, $18 \%$ de indivíduos com perfilhos. Esse resultado foi observado em áreas de floresta Atlântica, com diferente tempo de perturbação, em relação à floresta primária, e destaca que a perturbação aumenta o número de indivíduos perfilhados.

A capacidade de rebrota de algumas espécies observadas em áreas de restinga traz para esse ecossistema uma vantagem para a regeneração da área, uma vez que os indivíduos perfilhados persistem e aumentam o tamanho populacional, depois de perturbações no ecossistema. Este fator provavelmente irá afetar as características estruturais do ambiente recolonizado (Kauffman, 1991; Assumpção \& Nascimento, 2000; Sá, 2002; Cirne et al., 2003). Estudos em florestas tropicais têm demonstrado que a estrutura e a composição de espécies podem variar com relação aos nutrientes do solo (Laurance et al., 1999), e que diferentes espécies respondem de diferentes maneiras à variação desses nutrientes (Furtini Neto et al., 1999; Moreno \& Schiavani, 2001).

\section{Conclusão}

$\mathrm{O}$ estudo evidenciou que os tabuleiros costeiros apresentam heterogeneidade na estrutura espacial da vegetação e na diversidade, o que pode ser explicado pela associação entre as diferenças na composição florística, influência dos ecossistemas circunvizinhos (como a floresta atlântica, a restinga e a caatinga), e os processos de dispersão e extinção, que acabam influenciando na distribuição de espécies, em diferentes gradientes ecológicos, em escala regional e local. Fatores edáficos e graus 
de perturbação da área também são fatores que influenciam na composição florística.

Estudos posteriores, focados nos aspectos ecológicos (e.g. formas de vida, síndrome de dispersão, estratégias de regeneração) das espécies que compõem os tabuleiros costeiros, podem prover maior suporte para os resultados encontrados nesse estudo, além de possibilitar maior compreensão sobre a distribuição e a organização espacial das comunidades que integram o complexo da floresta Atlântica.

\section{Agradecimentos}

Agradecemos à Fundação O Boticário de Proteção à Natureza pelo financiamento do projeto "Caracterização florística-estrutural das restingas e tabuleiros do litoral do Nordeste brasileiro"; aà Coordenação de Aperfeiçoamento de Pessoal de Nível Superior (CAPES) e ao Conselho Nacional de Desenvolvimento Científico e Tecnológico (CNPq), que possibilitaram a execução da pesquisa.

\section{Referências}

Almeida Jr., E. B. 2006. Fisionomia e estrutura da restinga da RPPN Nossa Senhora do Outeiro de Maracaípe, Ipojuca, Pernambuco. 96f. Dissertação (Mestrado em Botânica), Universidade Federal Rural de Pernambuco, Recife.

Almeida Jr., E. B.; Olivo, M. A.; Araújo, E. L.; Zickel, C. S. 2009. Caracterização da vegetação de restinga da RPPN de Maracaípe, Pernambuco, com base na fisionomia, flora, nutrientes do solo e lençol freático. Acta Botanica Brasilica, 23, (1), 36-48. doi: 10.1590/S0102-33062009000100005

Andrade-Lima, D. 1954. Contribution to the study of the Flora of Pernambuco, Brazil. Monografia.Universidade Federal Rural de Pernambuco. Recife.

Andrade-Lima, D. 1960. Estudos fitogeográficos de Pernambuco. Arquivos do Instituto de Pesquisas Agronômicas, 5, 305-341.

Andrade-Lima, D. 1970. Recursos vegetais de Pernambuco. Boletim Técnico Instituto Agronômico, 41, 1-31.

Akatov, V.; Chefranov, S.; Akatova, T. 2005. The relationship between local species richness and species pool: a case study from the high mountains of the Greater Caucasus. Plant Ecology, 181, 9-22.

APG IV. 2016. An update of the Angiosperm Phylogeny Group classification for the orders and families of flowering plants: APG IV. Botanical Journal of the Linnean Society, 181, 1-20. doi.org/10.1111/boj.12385
Assumpção, J.; Nascimento, M. T. N. 2000. Estrutura e composição florística de quatro formações vegetais de restinga no complexo lagunar Grussaí/Iquipari, São João da Barra, RJ, Brasil. Acta Botanica Brasilica 14, (3), 301-315.

Barbosa, M. R. V. 1996. Estudo florístico e fitossociológico da Mata do Buraquinho, remanescente da Mata Atlâmtica em João Pessoa, PB. Tese de Doutorado, Universidade Estadual de Campinas, Campinas, Brasil.

Bigarella, J. J. 1975. The Barreiras Group in Northeastern Brazil. Anais da Acadêmia Brasileira de Ciências, 47, 465-410 (Suplemento).

Cantarelli, J. R. R.; Almeida Jr., E. B.; SantosFilho, F. S.; Zickel, C. S. 2012. Descrição da estrutura de uma vegetação de restinga da Área de Proteção Ambiental (APA) de Guadalupe, litoral sul de Pernambuco. In: ElDeir, A. C. A.; Moura, G. J. B.; Araújo, E. L. (Orgs). Ecologia e conservação de ecossistemas no Nordeste do Brasil. Recife, PE: NUPEEA. pp. 49-68.

Carvalho, G. M. 2000. Influência de processos estocásticos sobre a estruturação de comunidades em Floresta de Tabuleiros, Bahia, Brasil. Dissertação de Mestrado, Universidade Estadual de Santa Cruz, Ilhéus, Brasil.

Cestaro, L. A.; Soares, J. J. 2004. Variações florística e estrutural e relações fitogeográficas de um fragmento de floresta decídua no Rio Grande do Norte, Brasil. Acta Botanica Brasilica, 18, (2), 203-218. doi.org/10.1590/S0102-33062004000200001

Cirne, P.; Zaluar, H. L. T.; Scarano, F. R. 2003. Postifire resprouting on a sandy spit in a Brazilian coastal plain. Ecotropica, 9, 33-38.

Condit, R.; Pitman, N.; Leigh Jr., E. G.; Chave, J.; Terborgh, J.; Foster, R. B.; Núñez, V. P.; Aguilar, S.; Valencia, R.; Villa, G.; MullerLandau, H. C.; Losos, E.; Hubbell, S. P. 2002. Beta-diversity in tropical forest trees. Science, 295, 666-669.

Cottam, G.; Curtis, J. T. 1956. The use of distance measures in phytosociological sampling. Ecology, 37, 451-460.

Dias, A. T. C.; Zaluar, H. T.; Ganade, G.; Scarano, F. R. 2005. Canopy composition influencing plant patch dynamics in Brazilian sandy coastal plain. Journal Tropical Ecology, 21, 343-347.

Dunphy, B. K.; Murphy, P. G.; Lugo, A. E. 2000. The tendency for trees to be multiplestemmed in tropical and subtropical dry 
forests: studies of Guanica forest, Puerto Rico. Tropical Ecology, 41, (2), 161-167.

EMBRAPA. 1999. Sistema brasileiro de classificação de solos. EMBRAPA/CNPS. Rio de Janeiro, Brazil.

EMBRAPA. 2017. Manual de métodos de análise de solo, 3ed. Ministério da Agricultura, Pecuária e Abastecimento, Brasilia, DF. 573p.

Furtini Neto, A. E.; Resende, A. V.; Vale, R. V.; Faquin, V.; Fernandes, L. A. 1999. Acidez do solo, crescimento e nutrição mineral de algumas espécies arbóreas, na fase de muda. Cerne, 5, (2), 1-12.

Guedes, M. L. S. 1998. A vegetação fanerogâmica da reserva Ecológica de Dois Irmãos. In: Machado, I. C. S.; Lopes, A. V.; Porto, K. C. (eds.). Reserva Ecológica da Mata de Dois Irmãos: estudos em uma remanescente de Mata Atlântica em área urbana (Recife Pernambuco - Brasil). Recife, Editora Universitária. pp. 157-172.

Hitimana, J.; Kiyiapi, J. L.; Njunge, J. T. 2004. Forest structure characteristics in disturbed and undisturbed sites of Mt. Elgon Moist Lower Montane Forest, western Kenya. Forest Ecology and Management, 194, 269291.

IBGE. 1985. Atlas Nacional do Brasil: Região Nordeste. IBGE. Rio de Janeiro.

Ivanauskas, N. M.; Monteiro, R.; Rodrigues, R. R. 2004. Estrutura de um trecho de floresta amazônica na bacia do alto rio Xingu. Acta Amazônica, 34, (2), 281-305.

Kauffman, J. B. 1991. Survival by sprouting following fire in tropical forests of the eastern amazon. Biotropica, 23, (3), 219-224.

Krebs, C. J. 1989. Ecological methodology. Harper Collins Publishers, Massachusetts. USA.

Laurance, W. F.; Fearnside, P. M.; Laurance, S. G.; Delamonica, P.; Lovejoy, T. E.; Merona, J. M. R.; Chambers, J. Q.; Gascon, C. 1999. Relationship between soils and Amazon forest biomass: a landscape-scale study. Forest Ecology and Management, 118, 127138.

Mabesoone, J. M.; Campos, E.; Silva, A.; Beurlen K. 1972. Estratigrafia e origem do grupo Barreiras em Pernambuco, Paraíba e Rio Grande do Norte. Revista Brasileira de Geociências, 2, (3), 173-188.

Magalhães, J. H. R. 2018. Estrutura da comunidade arbórea de um remanescente de floresta madura nos tabuleiros costeiros do extremo sul da Bahia, Brasil. Bol. Mus. Biol. Mello Leitão, 40, (2), 93-122.
Martins, F. R. 1991. Estrutura de uma floresta mesófila. Editora Unicamp. Campinas.

MMA/IBAMA - Ministério do Meio Ambiente/ Instituto Brasileiro do Meio Ambiente e dos Recursos Naturais Renováveis. 2003. Plano de manejo da Reserva Biológica Guaribas. Brasília: CHESF, MRS Estudos Ambientais, 520p.

Moreno, M. I. C.; Schiavini, I. 2001. Relação entre vegetação e solo em um gradiente florestal na Estação Ecológica do Panga, Uberlândia (MG.). Revista Brasileira de Botânica, 24, (4), 537-544.

Neves, S. M. 1993. Análise Geo-ambiental do Litoral Sul da Paraíba: Pitimbú-Caaporã. Recife. Mestrado em Geografia. Universidade Federal de Pernambuco. 137p.

Oliveira Filho, A. T.; Fontes, M. A. L. 2000. Patterns of floristic differentiation among Atlantic Forest in south-eastern Brazil, and the influence of climate. Biotropica, 32, 793 810.

Oliveira, R. R. 2002. Ação antrópica e resultantes sobre a estrutura e composição da Mata Atlântica na Ilha Grande, RJ. Rodriguésia, 53, (82), 33-58.

Oliveira-Filho, A. T. 1993. Gradient analysis of an area of coastal vegetation in the state of Paraíba, Northeastern Brazil. Edinburgh Journal of Botany, 50, (2), 217-236.

Parmentier, I.; Stévart, T.; Hardy, O. 2005. The inselberg flora of Atlantic Central Africa. I. Determinants of species assemblages. Journal of Biogeography, 32, 685-696.

Phillips, O. L.; Vargas, P. N.; Agudo, A. L. M.; Cruz, A. P.; Zans, M. E. C.; Sánchez, W. G.; Yli-Halla, M.; Rose, S. 2003. Habitat association among Amazonian tree species: a landscape-scale approach. Journal of Ecology, 91, 757-773.

Pitman, N. C. A.; Terborghm, J. W.; Silman, M. R.; Núñez, P. V.; Neill, D. A.; Cerón, C. E.; Palacios, W. A.; Aulestia, M. 2001. Dominance and distribution of tree species in upper Amazonian terra firme forests. Ecology, 82, (8), 2101-2117.

Prates, M.; Gatto, L. C. S.; Costa, M. I. P. 1978. Geomorfologia In: Brasil-Projeto RADAMBRASIL. Folhas SB. 24/25 Jaguaribe/Natal. Projeto RADAMBRASIL, Rio de Janeiro, pp. 301-339.

Ranzani, G.; Freire, O.; Kinjo, T.; Cesar, C. M. 1964. Considerações gerais sobre os solos de tabuleiro do Nordeste. Boletim de Recursos Naturais, 2, (1), 45-70.

Roth, L. C. 1999. Anthropogenic change in subtropical dry Forest during a century of 
settlement in Jaiquí Picado, Santiago Province, Dominican Republic. Journal of Biogeography, 26, 739-759.

Sá, C. F. C. 2002. Regeneração de um trecho de floresta de restinga na Reserva Ecológica Estadual de Jacarepiá, Saquarema, Estado do Rio de Janeiro: II - Estrato arbustivo. Rodriguésia, 53, (82), 5-23.

Salgado, O. A.; Jordy Filho, S.; Gonçalves, L. M. C. 1981. Vegetação In: Projeto RADAMBRASIL. Folhas SB. 24/25 Jaguaribe/Natal. Projeto RADAMBRASIL, Ministério das Minas e Energia, Rio de Janeiro, Brasil. pp. 485-535.

Santos-Filho, F. S.; Zickel, C. S. 2013. Origem e estrutura da costa e vegetação de restinga: o caso do litoral do Piauí. In: Santos-Filho, F. S.; Soares, A. F. C. L.; Almeida Jr., E. B. (Org.). Biodiversidade do Piauí: pesquisa \& perspectivas Vol. 2. 1ed. Curitiba: Editora CRV, 2, pp. 11-36.

Scarano, F. R. 2002. Structure, function and floristic relationships of plant communities in stressful habitats marginal to the Brazilian Atlantic rainforest. Annals of Botany, 90, 517-524.

Shepherd, G. J. 2009. Fitopac 2: manual do usuário Programa Fitopac 2: Campinas, UNICAMP.

Tavares, S. 1960. Estudos geobotânicos no Rio Grande do Norte. Arquivos do Intituto de Pesquisa Agronômicas, 5, 39-51.

Tavares, S. 1964. Contribuição ao estudo da cobertura vegetal dos tabuleiros do Nordeste. Boletim de Recursos Naturais, 2, (1/4), 13-25.
Vicente, A. 2006. Caracterização floristicas dos tabuleiros litorâneos do nordeste do Brasil. 100f. Tese (Doutorado em Botânica), Universidade Federal Rural de Pernambuco.

Watson, D. M. 2002. A conceptual framework for studying species composition in fragments, islands and other patchy ecosystems. Journal of Biogeography, 29, 823-834.

Sousa, J. L. M.; Santos-Filho, F. S. 2020. Estudos Botânicos nos Tabuleiros Litorâneos do Brasil. Revista Brasileira de Geografia Física, 13, (3), 1335-1347. doi.org/10.26848/rbgf.v13.3.p1335-1347

Weaver, P. L.; Chinea, J. D. 2003. Secondary subtropical dry Forest at the La Tinaja Tract of the Cartagena Lagoon National Wildlife Refuge, Puerto Rico. Caribbean Journal of Science, 39, (3), 273-285.

Zar, J. 1999. Biostatistical Analysis. 4th ed. Prentice Hall, New Jersey, USA.

Zickel, C. S.; Santos, A. V.; Almeida Jr., E. B.; Tabarelli, M. 2012. Estrutura e riqueza de espécies lenhosas em áreas de tabuleiro arenoso do Rio Grande do Norte, Nordeste do Brasil. In: El-Deir, A. C. A.; Moura, G. J. B.; Araújo, E. L. (orgs). Ecologia e conservação de ecossistemas no Nordeste do Brasil. Recife, PE: Nupeea. pp. 69-88.

Zickel, C. S.; Vicente, A.; Silva, S. S. L.; SantosFilho, F. S.; Soares, C. J. R. S.; Almeida Jr., E. B. 2015. Vegetação lenhosa de uma restinga em Pernambuco: descrição estrutural e similaridade. Pesquisas Botânica, 68, 271285. 